\title{
THE SUBDIVIDED DRIVING SYSTEM USED IN STEPPING MOTOR
}

\author{
Run $M a^{1}$ \\ ${ }^{1}$ Chongqing Industry Polytechnic College, Chongqing, 401120, China. \\ Email:maruneducq@yeah.net
}

\begin{abstract}
To study the application of subdivided driving system in stepping motor, improve the resolution of stepping motor, and make the stepping motor run smoothly, the hybrid stepping motor (model 42BYG250-40) was taken as the basic research object. In addition, the mathematical model of two-phase hybrid stepping motor was established, and the subdivided driving circuit of stepping motor supporting the system was designed. Moreover, the STM32F103ZET6-centerted control unit, the TC1005-centerted drive unit stepping motor driving chip, and the H-bridge power amplifier circuit were designed. Furthermore, the stepping motor subdivided driving simulation model was simulated using Matlab / Simulink tool. With the increase of the subdivision number, the oscilloscope needs to choose a larger time interval to see the complete waveform. In 2-subdivision, the current of stepping motor winding is in obvious step shape. When the subdivision number is gradually increased, the output waveform of oscilloscope becomes smoother from step shape, and it is closer to sine wave. Meanwhile, the vibration and noise of stepping motor operation will be reduced. In 128-subdivision, the vibration and noise of motor has been very small, and there is no obvious change when increasing the subdivision number to 256-subdivision, which shows that the system can still run stably and accurately in 256-subdivision. Through the experimental analysis, the design can achieve 256-subdivision with high precision, and improve the low-frequency vibration and noise. It indicates that the running stability of the stepping motor is better, and the vibration and noise problems are also controlled to a certain extent. To sum up, the subdivided driving system can subdivide the current, enhance the stability of the motor, and achieve the expected design goal, which can provide theoretical support for the research and development of stepping motor.
\end{abstract}

Keywords: Stepping Motor; Two-Phase Hybrid Stepping Motor; Subdivided Driving System; Noise Improvement; Subdivided Current.

\section{Introduction}

Stepping motor is a control motor which transforms electric pulse signal into corresponding angular displacement or linear displacement through pulse signal conversion control technology. The comprehensive performance of the stepping motor, the structure of the stepping motor itself, and its driving technology. The closed step motor is also called pulse motor [1].

The frequency and number of electric pulse signal determine the speed and position of stepping motor [2].

With the rapid development of computer and embedded technology, stepping motor technology has been promoted. Because of its small size, high cost performance, precise control, and other advantages, stepping motor has become a widely used technology in the field of industrial automation.

As the driving mode of stepping motor is too traditional, the current widely used stepping motor is still the whole-step or half-step operation mode, and special power supply is needed to provide power for driving. Under such driving mode, the stepping motor has the defects of low precision, large noise, and high error. Hence, to adapt to more diverse utilization environment and production needs and solve the problem of too large step angle and too low resolution when stepping motor is running, new stepping motor drive technology is studied, that is, subdivided driving technology.

However, since subdivided driving technology is not perfect, there is still a very large research space and application value. Additionally, the development of a multi-subdivision and high-precision stepping motor driving system not only improves the efficiency and quality of production and life, but also improves the level of science and technology in China.

It also has high economic value and great practical significance.

The traditional stepping motor driving is determined by the ring distributor. By controlling the current flowing into each phase winding of the stepping motor, the rotating composite magnetomotive force of the stepping motor drives 
the rotor to rotate step by step. Through this driving, the stepping motor has only a whole-step and a halfstep operation mode, so there are only two kinds of step angles [3-5].

With the rapid development of stepping motor driving technology, subdivided driving technology solves the shortcomings of large step angle, inaccurate control, low-speed vibration, and large noise of stepping motor, enhances the resolution of stepping motor, and is conducive to the smooth operation of stepping motor.

The hybrid stepping motor 42BYG250-40 is taken as the basic research object, and the mathematical model of two-phase hybrid stepping motor is established; the subdivided driving circuit of the stepping motor is designed to support the control and drive of the system.

The hardware circuit design mainly includes three parts, the system control circuit, the driving circuit, and the peripheral function circuit.

The control circuit is the control unit with STM32F103ZET6 as the core; the drive circuit is the drive unit with TC1005 stepping motor driving special chip as the core, and the H-bridge power amplifier circuit is designed to drive the stepping motor.

The peripheral function circuit is mainly used for the serial port circuit to communicate with the computer, joint test action group (JTAG) circuit for program debugging, and optocoupler isolation circuit and power supply circuit for isolating control module and driving module. The software is designed according to the layered structure proposed by the main controller software interface standard.

The stepping motor subdivided driving simulation model is carried out by using Matlab/Simulink simulation tool.

\section{Method}

The hybrid stepping motor is mainly composed of stator parts, rotor parts, casing, and end cover.

The stator parts include stator core, winding, and insulating materials. The stator core is made of nondirectional silicon steel sheets. The rotor parts are composed of rotor core, permanent magnet material, and shaft. Rotor core is usually made of silicon steel sheet, block electrical steel, or powder metallurgy material. The rotor is composed of a ring-shaped magnetic steel and two iron cores. The ring-shaped magnetic steel is in the middle of the rotor and is magnetized axially. The two iron cores are respectively installed at both ends of the magnetic steel, so that the rotor is axially divided into two magnetic poles. The casing mainly strengthens the rigidity of the motor, protects the motor, and forms part of the magnetic circuit of the stator core. The end cover supports the rotor to ensure the air gap.

\subsection{Subdivided driving technology}

Subdivided driving control technology is also called micro-step control technology. The essence of this technology is to input step-by-step change direction current into the winding of the stepping motor, so that the step angle can be reduced when each step rotates, thereby improving the resolution of the motor [6-8]. The subdivided driving control technology reduces the step angle of each step of the motor, improves the inherent resolution of the motor, and has a good restraining effect on the low frequency oscillation, step loss, and large noise that are common in most motors. It is the novel control driving mode specially designed for solving the problems of low accuracy, motor oscillation, and large noise of the stepping motor [9].

The basic principle of subdivision transforms the current flowing through the motor winding from the previous rectangular waveform into a stepped wave shaped like a sine wave, and to add a number of continuously changing intermediate values between 0 and $I_{\max }$. The corresponding magnetic field vector will generate several continuously changing composite magnetic field vectors, and the angle between the composite magnetic field vectors is the step angle. After adding the magnetic field vector, the step angle will be smaller, so that the goal of subdivision can be achieved. For any phase stepping motor, the step angle is set to be $\theta_{\mathrm{e}}$ when it moves in the whole-step mode. After subdividing, the step angle becomes $\theta_{\mathrm{e}} / \mathrm{d}$, where $\mathrm{d}$ represents the subdivision number, that is, the original step is $\theta_{\mathrm{e}}$, now the step is $\theta_{\mathrm{e}}$, and the total step is $\theta_{\mathrm{e}} / \mathrm{d}[10]$.

Taking the two-phase hybrid stepping motor as an example, when it moves in the whole-step mode, its stator winding is electrified in the order of $A \rightarrow B \rightarrow(-A) \rightarrow(-B) \rightarrow A$ (assuming that only phase $A$ winding is electrified) [11] (Figure 1).

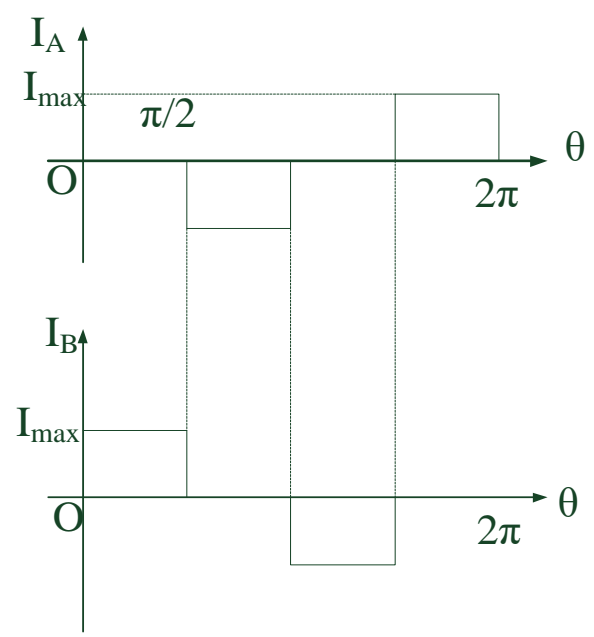

Figure 1: Two-phase motor: A and B phase current

In fact, the subdivided driving control of stepping motor is not the traditional open-loop control, but 
the closed-loop control of phase winding current which tracks the given current waveform.

The principle of subdivided driving closed-loop control is that the state of the input control pulse sequence is counted by the cycle addition and subtraction counter, and the given current value is stored in EPROM.

The control circuit converts the digital value of the current of each phase winding into the analog value in real time through the $\mathrm{D} / \mathrm{A}$ module according to the given current waveform and the state of the input control pulse sequence stored in advance.

The current error value is obtained by comparing the current value analog value with the current feedback value. The error value changes the driving pulse width of the main electric MOSFET through the action of the current controller and the pulse width modulation (PWM) control unit, and then controls the phase winding current of the stepping motor to maintain a constant value [12,13] (Figure 2).

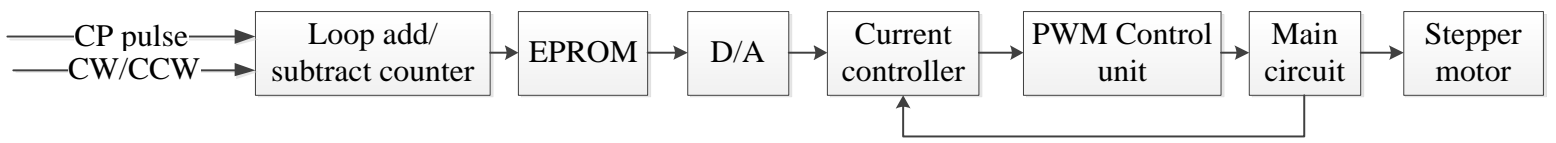

Figure 2: Basic structure block diagram of stepping motor subdivided driving circuit

Figure 3 shows the realization process of replacing the sine wave with $\mathrm{PWM}$ wave, in which (a) is the sine half wave and (b) is the pulse sequence. The sine half wave is regarded as n-equal pulse of equal width and unequal amplitude, and the amplitude of these pulses changes according to the sine law. These equal width and unequal amplitude pulse sequences are replaced by rectangular pulse sequences with equal frequency and unequal width.

Then, these pulse sequences with equal frequency and unequal width are PWM waveforms [14]. According to the principle of area equivalence, PWM waveform and sine half wave are equivalent.

For the negative half cycle of sine wave, the PWM waveform can be obtained by the same method.

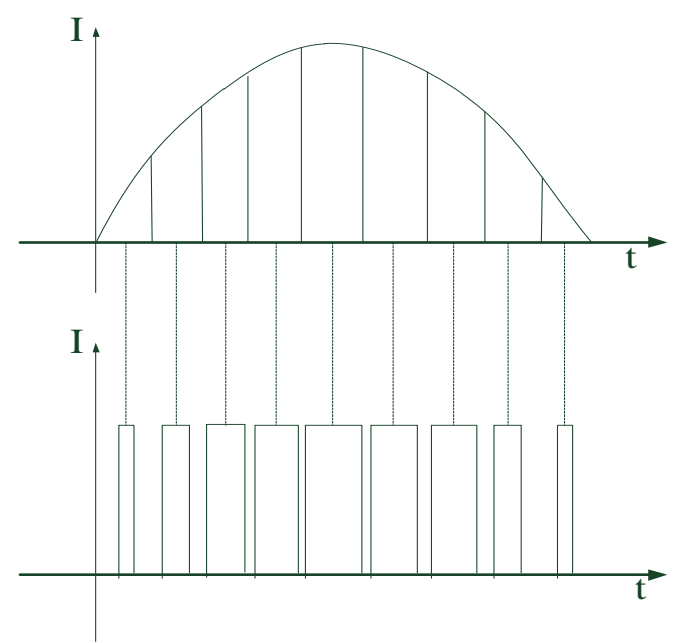

Figure 3: Corresponding diagram of sine wave and PWM wave

Using this method, the current of motor winding needs to be changed and there are Eq. 1 and Eq. 2:

$$
\begin{aligned}
& \mathrm{i}_{A}=I_{\text {max }} \sin \theta \\
& \mathrm{i}_{B}=I_{\text {max }} \cos \theta=I_{\text {max }} \sin \left(\theta+\frac{\pi}{2}\right)
\end{aligned}
$$

The difference between current $i_{A}$ and $i_{B}$ is $2 / \pi$ (Figure 4).

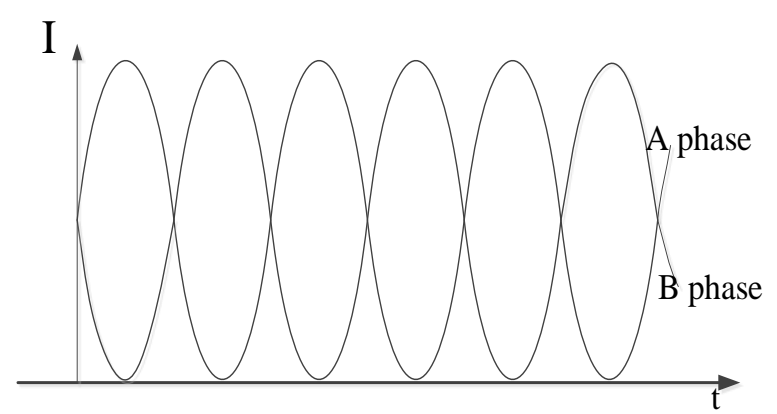

Figure 4: Location of currents $i_{A}$ and $i_{B}$

\subsection{System hardware design method}

The hardware design requirements of the system are as follows: first, Use Simulink, a visual simulation tool of Matlab, to complete the simulation of the twophase hybrid stepping motor subdivided driving model; second, design the hardware circuit to support the system requirements; third, design and debug the software program to meet the system requirements, including the stepping motor subdivision driver and the serial communication program between the lower computer and the upper computer; fourth, realize the real-time communication between the upper computer and the subdivision drive system of stepping motor; fifth, design and realize 256-subdivision high-precision stepping motor driving system.

The hardware scheme design of the system is an important part of the overall scheme design of the system. The system hardware includes optocoupler isolation, power amplifier circuit, H-bridge circuit, current protection circuit, and FPGA core board (Figure 5). 


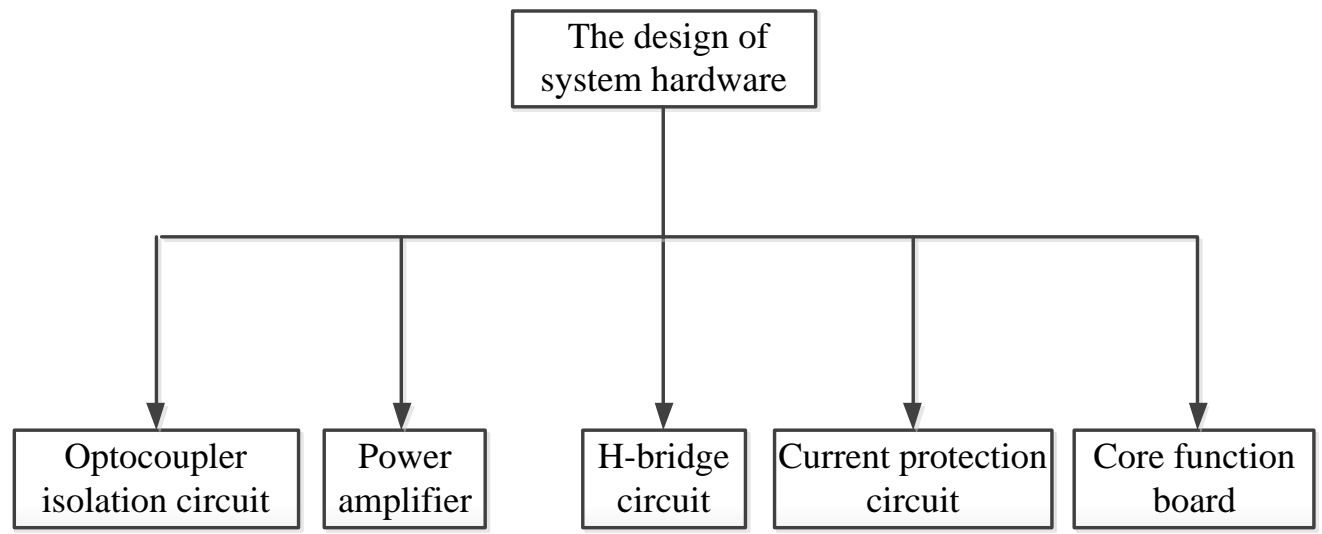

Figure 5: System hardware design

The processor of the control part of the system adopts the enhanced STM32F103ZET6 microprocessor of STM32f103x series, which can meet the needs of the system. The system uses serial peripheral interface communication to realize the data communication and processing between STM32 and the stepping motor driver chip TC1005, as well as the data communication with the upper computer. In the system design, all I / O ports of STM32 chip have been set with outgoing ports, which is convenient for later function expansion or secondary development of the system.

\subsection{System software design method}

According to the basic theory of subdivision, the system will adopt the subdivided driving method which combines the uniform rotation of current vector and the sinusoidal PWM (SPWM). Because the A-phase and B-phase currents generated by this method have a phase difference of $\pi / 2$, a ROM table should be created to store the required sinusoidal current values. The essence of subdivided stepping motor is subdivided current, so it is necessary to create a current sub-divider and PWM converter to subdivide stepping motor. The function of PWM converter is to change sine wave of different size into rectangle wave of different width, and drive peripheral hardware circuit with rectangle wave of different width, thus achieving the purpose of subdivided stepping motor. For this reason, the design scheme of FPGA-based subdivided driving system is designed.

The scheme mainly includes: subdivision selection, address selection, dual-port memory, analog-to-digital conversion, current feedback, current value conversion, PWM conversion, dead zone compensation, and direction conversion.

Figure 6 shows the design scheme of SPWM subdivided driving system.

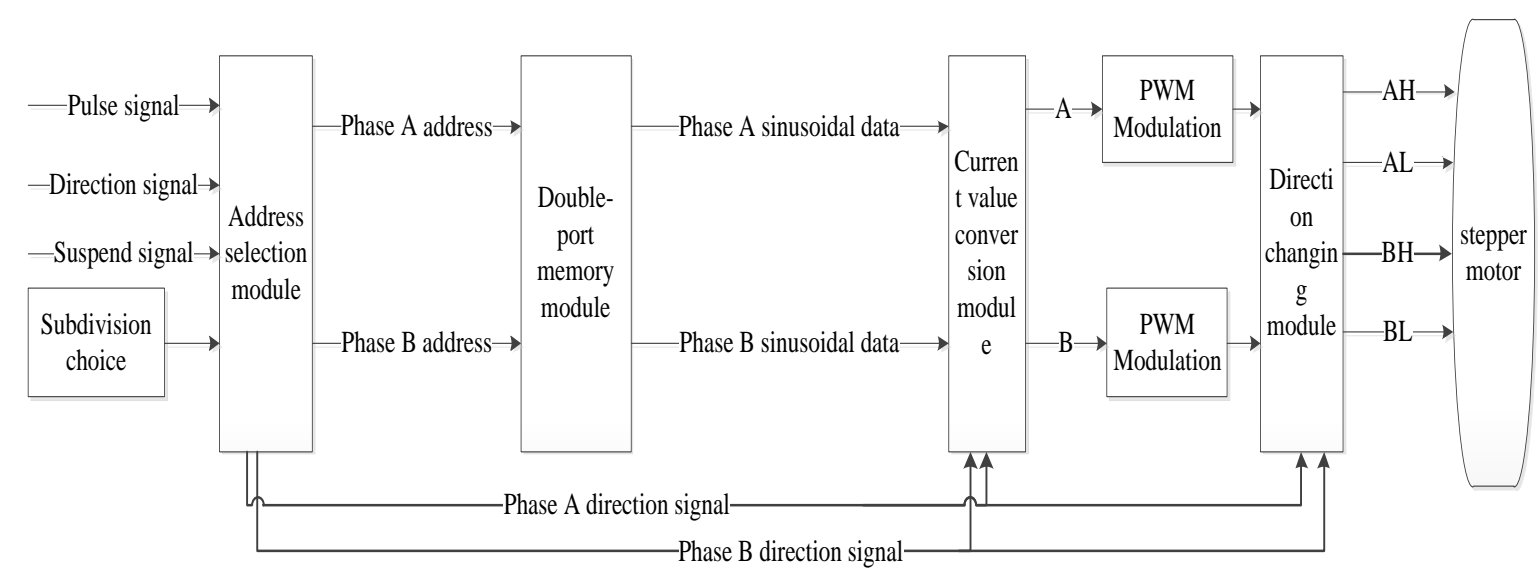

Figure 6: SPWM subdivided driving system design plan

In the design of subdivided driving system, the subdivision selection module determines the number of subdivision selection first. When the pause signal is equal to 0 and the direction signal is equal to 1 , the address selection module will add (subtract) 1 to the input pulse signal according to the subdivided number determined by the subdivision selection module, and store the memory data with the phase difference of $\pi / 2$ as the address of phase

$A$ and phase B in the dual-port memory module.

There are 1024 binary sinusoidal current values in the dual-port memory, which are output sinusoidal current values of phase A and phase B after the address selection module. However, the 
data in the dual-port memory are all signed current values, and what is needed is the binary number of the unsigned bits. At this time, the current value conversion module is used, which can transform the current value data of the unsigned bits according to the current values of the directed A and B phases.

Then, according to the current value data of phase A and phase B output by the current value conversion module, the width of the output rectangular wave is adjusted, and the proportion of output 0 and 1 is adjusted to complete the function of the PWM module. The function of the direction conversion module is to adjust the direction of the PWM output signal (adjust the current direction): in the range of $0 \sim \pi$, the PWM signals output by the direction conversion module are $\mathrm{AH}$ and $\mathrm{BH}$, while in the range of $\pi \sim 2 \pi$, the PWM signals output by the direction conversion module are AL and BL [15].

The output AH AND AL are used as the input of the peripheral hardware circuit, and then the output of the hardware circuit drives the stepping motor.

The main software compiling environment for the design and implementation of this system is the $\mathrm{u}$ Vision5 IDE, and the software version is $\mathrm{u}$ Vision V5.14.0.0.

This development platform provides a visual $\mathrm{C} / \mathrm{C}++$ integrated development environment for the embedded processor STM32.

The interface is clear, concise, and easy to understand. In the running process of the program, the single-step operation is adopted to record every step of the application program, and the application program can be analyzed in real time.

The main program is used to initialize clock, constant, and variable, register, I/O port, open interrupt and infinite loop. Figure 7 shows the software flow of the main program. At the beginning of the program, the system clock, constants, and variables are initialized first, then the interrupt flag is initialized and the interrupt is turned off.

The peripheral is configured, then the interrupt is turned on, and finally the program enters the main program and waits for the interrupt until it occurs. After an interrupt occurs, the system responds to the interrupt by jumping out of the main loop and executing the corresponding interrupt service subprogram. After the execution of the interrupt service subprogram, the system returns to the main loop and waits for the next interrupt again.

No specific program is executed in the main cycle, and all functions such as subdivision program, data communication, PWM pulse, overvoltage, and overcurrent protection are realized in the interrupt service program.

In addition, watchdog code is set to keep the program stable and not prone to errors, thereby improving the reliability of program operation.

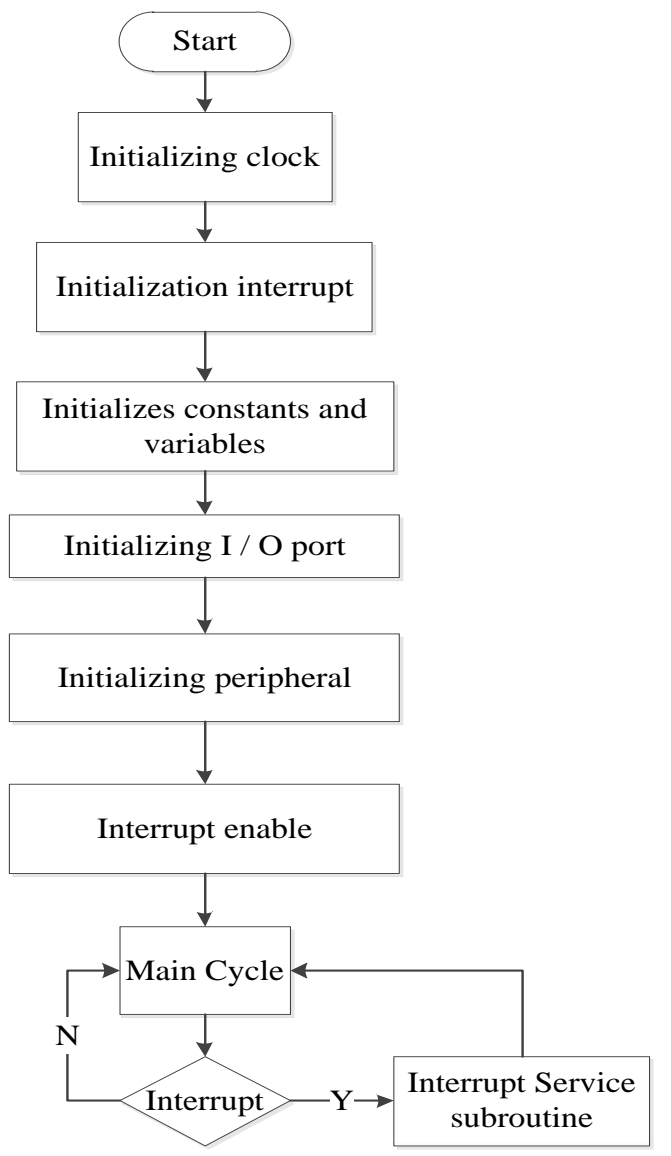

Figure 7: Flow chart of system main program design

\subsection{System simulation method}

To verify the correctness of the stepping motor subdivided driving system designed, Matlab/Simulink software is used to simulate the stepping motor subdivided driving system.

The software version is R2016b (9.1.0.441655). A two-phase hybrid stepping motor subdivided driving system simulation model is built. Matlab is the highperformance numerical calculation software of Math Work company. Simulink based on Matlab platform is a visual integrated simulation environment in the field of dynamic simulation. It is often used to model, simulate, and analyze the problems solved.

The Simulink library contains a rich module for control system design and simulation.

The developers only need to modify the parameters and attributes of the modules to establish the simulation model, and then observe the simulation process and experimental results through the oscilloscope module.

The simulation model includes two-phase hybrid stepping motor model, H-bridge model, pulse generation module, and subdivision module.

The stepping motor model provided in Simulink library is a package module.

The stepping motor used in this system is a twophase hybrid stepping motor. The number of rotor teeth is 50 and the step angle is $1.8^{\circ}$. 


\section{Results}

\subsection{PWM waveform test results}

During the simulation, set the pulse frequency to 2500 pulse / s, and simulate the model with 2, 4, 8, 16, 32, 64, 128 and 256 subdivisions, respectively. To test whether the function of FPGA module can be realized, use the probe of oscilloscope to connect the output pin of FPGA to test the waveform.

At this time, the set system clock frequency $\mathrm{clk}=50 \mathrm{MHz}$.

Figures 8 and 9 show the observed 8-subdivision and 16-subdivision square wave signals.

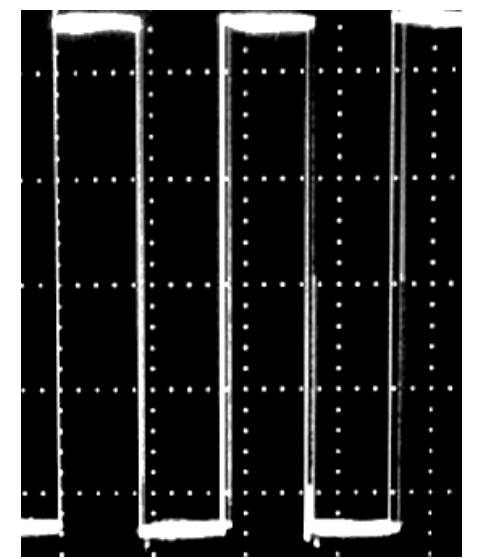

Figure 8: 8-subdivision square wave signal

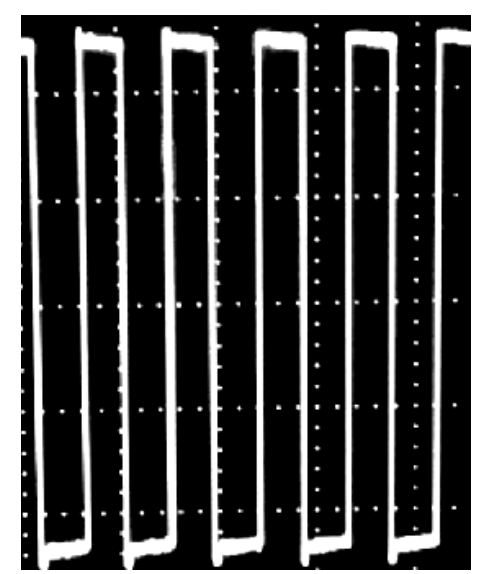

Figure 9: 16-subdivision square wave signal

The comparison between the two waveforms shows that, with the increase of the number of subdivisions, the width of the waveforms will become smaller, the number of subdivision step angle will be more, and the step angle will be smaller, which is consistent with the scheme design.

\subsection{Experimental results of one-way winding current}

The equipment needed for the system experimental verification includes switching power supply, system control drive board, two-phase fourwire hybrid stepping motor, multi-meter, and oscilloscope. The maximum current is $2.8 \mathrm{~A}$, the step angle is $1.8^{\circ}$, and the torque is $1.2 \mathrm{~N} . \mathrm{m}$; the switch power supply is selected to output $24 \mathrm{~V}$ direct current power supply.

During the experiment, the upper computer sends the control signal to the control unit, and STM32 executes the corresponding function to drive the step motor to realize the corresponding operation after receiving the data command.

A resistor with a resistance of $1 \Omega$ and a power of $3 \mathrm{~W}$ is connected in series between the output end of the driving system and the phase winding of the stepping motor. The $\mathrm{CH} 1$ probe of the oscilloscope is connected to both ends of the resistor to measure the voltage. The waveform of the voltage approximately represents the waveform of the phase winding current. Meantime, the $\mathrm{CH} 2$ probe of oscilloscope is used to measure the input pulse. The output voltage waveforms of 8 subdivision, 16 subdivision, 32 subdivision, 64 subdivision, 128 subdivision, and 256 subdivision are tested respectively when the input pulse signal is kept at $100 \mathrm{~Hz}$.

After connecting the controller, FPGA board, hardware circuit, power supply, and oscilloscope, and downloading the program to FPGA development board, connect the power supply.

Figures 10 and 11 show the measured sinusoidal current of 8-subdivision and 16 subdivision one-way winding, respectively. With the increase of the subdivision number, the winding current of the motor becomes more sinusoidal, the waveform becomes more stable, and the motor runs more smoothly.

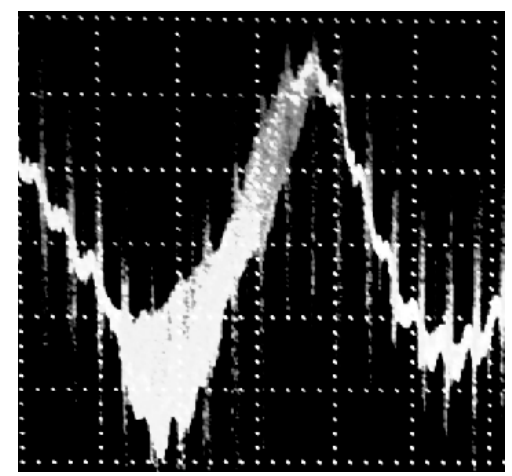

Figure 10: 8-subdivision sinusoidal current

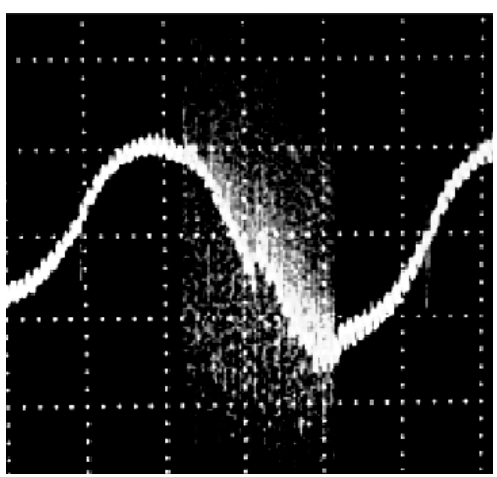

Figure 11: 16-subdivision sinusoidal current 
The test results of the physical experiment platform are basically consistent with the simulation results, which shows that the system designed and implemented here is feasible and effective. With the increase of the subdivision number, the oscilloscope needs to choose a larger time interval to see the complete waveform. In 2-subdivision, the current of stepping motor winding is in obvious step shape. When the subdivision number is gradually increased, the output waveform of oscilloscope becomes smoother from step shape, and it is closer to sine wave. Additionally, the vibration and noise of stepping motor will decrease with the operation. In 128-subdivision, the vibration and noise of motor has been very small, and there is no significant change when increasing the subdivision number to 256. It shows that the system can still operate stably and accurately in 256-subdivision. Through experimental analysis, the design can achieve high precision 256-subdivision, and improve the lowfrequency vibration and noise better.

It indicates that the running stability of stepping motor is better, and the vibration and noise problems are also controlled to a certain extent (Figure 12).

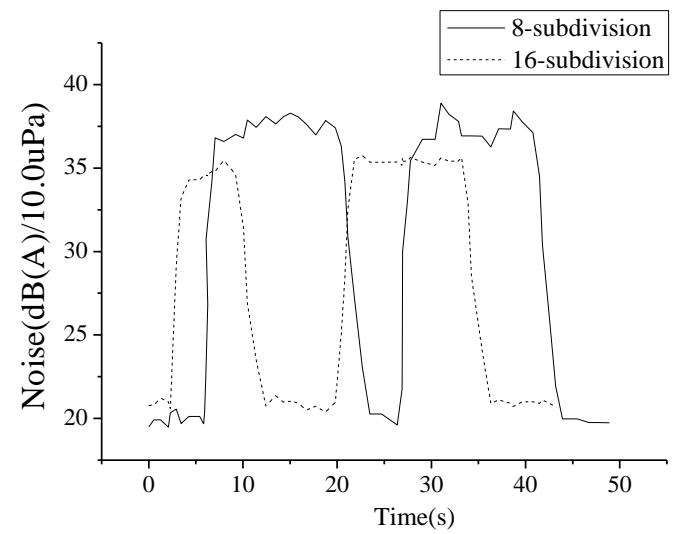

Figure 12: Comparison of 8-subdivision and 16subdivision noises

\section{Discussion}

The application of subdivided driving technology in stepping motor is discussed. The subdivided driving control technology reduces the step angle of each step of the motor by using the current step in method and has a better inhibition effect on the lowfrequency oscillation, step loss, and large noise that are common in most motors.

Based on the analysis of the mathematical model of the stepping motor, a two-phase hybrid stepping motor subdivided driving simulation model is built. The main work of the model building is to build the subdivision module according to the subdivision drive principle. To debug and simplify the simulation model interface, the built micro-molecule system is encapsulated. Finally, the pulse signal waveform of the drive system, the given subdivision current waveform, and the current waveform input to the phase winding of the stepping motor are tested by the oscilloscope. Using the visual simulation tool Simulink of Matlab, the simulation of the two-phase hybrid stepping motor subdivision drive model is completed. The hardware circuit supporting the system requirements is designed, and the software program meeting the system requirements is designed and debugged. The real-time communication between the upper computer and the stepping motor subdivided driving system is realized, and the high-precision stepping motor drive system with 256 subdivision is designed and realized.

In addition, a hardware circuit which integrates control and drive is designed to meet the requirements of the system. The hardware circuit of the system mainly includes the control part circuit, the drive part circuit, and the peripheral function circuit. By consulting the data and comparing, the chip selection is finally determined, and the STM32F103ZET6 chip is selected as the system microprocessor to design the minimum system.

TC1005 is selected as the stepping motor driver chip and FDD8424H as the switch tube of the $\mathrm{H}$ bridge.

TC1005 is an intelligent chip with built-in load and locked rotor detection function, which supports many subdivision numbers. FDD8424H is an N \& $\mathrm{P}$ dual channel MOSFET, which simplifies the hardware design, considers the stability of the test rate system, and takes isolation treatment for the system. To facilitate the system debugging and communication, JTAG circuit and serial to serial communication interface circuit are designed.

Moreover, considering the convenience of practical operation and application, the upper computer interface is developed by using Lab VIEW software to realize the intelligent control of stepping motor. Finally, based on hardware circuit, through the programming of software algorithm, the physical experiment platform is built successfully.

Through the comparison and analysis of the experimental platform test and model simulation results, the system achieves the expected goal.

The system designed and implemented is only completed in the laboratory, not in the actual application environment for a long time to track and test.

The follow-up work will be carried out for a specific application system; the design time in software debugging is limited and can be further optimized to make the code operate with high-speed.

Attention is also necessary to paid to further improve the stability of the system during operation, and in the driver hardware circuit, the circuit structure has the problem of high-power consumption, which needs to be improved. 


\section{Conclusions}

The test results of the physical experiment platform are basically consistent with the simulation results, which shows that the system designed and implemented is feasible and effective. With the increase of the subdivision number, the oscilloscope needs to choose a larger time interval to see the complete waveform. In 2-subdivision, the current of stepping motor winding is in obvious step shape.

When the subdivision number is gradually increased, the output waveform of oscilloscope becomes smoother from step shape, and closer to sine wave. Meanwhile, the vibration and noise of stepping motor operation will be reduced. In 128subdivision, the vibration and noise of motor has been very small, and there is no obvious change when the subdivision number continues to increase to 256-subdivision, suggesting that the system can still operate stably and accurately in 256subdivision.

The experimental analysis shows that the design can achieve 256-subdivision with high precision, and improve the low-frequency vibration and noise.

It shows that the running stability of the stepping motor is better, and the vibration and noise problems are also controlled to a certain extent.

The subdivided driving system can subdivide the current, enhance the stability of the motor, and achieve the expected design goal.

The subdivided driving technology can improve the stability of stepping motor and reduce noise.

Although the two-phase hybrid stepping motor subdivided driving system designed achieves the expected goal and has good operation effect, there are still some deficiencies in this system, especially in the future practical application environment, which still needs to be improved.

\section{References}

[1] Viaene J. D., Haemers M., Verbelen F, et al. (2018) Current Reduction in Stepping Motor Applications using an Adaptive PI controller based on Linearized Dynamics Research funded by a PhD grant of the Research Foundation Flanders (FWO), Belgium. 51(4), 107-112.

[2] Faber F., Thiennimitr P., Spiga L, et al. (2017) Respiration of Microbiota-Derived 1,2propanediol Drives Salmonella Expansion during Colitis. Plos Pathogens, 13(1), 1-11.

[3] Oliveira. A. S., Reiche. M. S., Vinescu. C. I, et al. (2018) The cognitive complexity of concurrent cognitive-motor tasks reveals age-related deficits in motor performance. Scientific Reports, 8(1), 20.
[4] Oliveira A. S., Reiche M. S., Vinescu C. I, et al. (2017) Development of a novel long range piezoelectric motor based on double rectangular trajectories driving. Microsystem Technologies, 24(12), 1-10.

[5] Liang X., Xiao X., Zhu Y, et al. (2018) Design and experiment on pot-seedling supplementing endeffector for vegetables in cotyledon phase. Nongye Gongcheng Xuebao/transactions of the Chinese Society of Agricultural Engineering, 34(9), 49-57.

[6] Dürr V., Theunissen L. M., Dallmann C. J, et al. (2018) Motor flexibility in insects: adaptive coordination of limbs in locomotion and nearrange exploration. Behavioral Ecology \& Sociobiology, 72(1), 15.

[7] Dos Santos A. S., De A. W., Popik B, et al. (2017) Characterization of a cerebral palsy-like model in rats: Analysis of gait pattern and of brain and spinal cord motor areas. 60, 48-55.

[8] Almarwani M., Van Swearingen J. M., Perera S, et al. (2017) The Effect of Auditory Cueing on the Spatial and Temporal Gait Coordination in Healthy Adults. Journal of Motor Behavior, 51(3), 1-7.

[9]Ding Y., Xiao X. (2017) Control Strategies of Flexible Load Driven Directly by Permanent Magnet Synchronous Motor. Diangong Jishu Xuebao/transactions of China Electrotechnical Society, 32(4), 123-132.

[10] Daouda M., Lin C. L., Lee C. S, et al. (2017) Model predictive control of sensorless hybrid stepper motors in auxiliary adjuster for stereotactic frame fixation. Mechatronics, 47, 160-167.

[11] Sattar M., Wei C. (2017) Modeling, Analysis and Testing of Micro Disturbances of Solar Array Drive Assembly. Applied Mechanics \& Materials, 865, 496-501.

[12] Gölcük A., Güler İ. (2017) The Use of Stepper Motor-Controlled Proportional Valve for Fio2 Calculation in the Ventilator and its Control with Fuzzy Logic. Journal of Medical Systems, 41(1), 1.

[13] Lempart M., Kügele M., Snäll J, et al. (2017) Development of a novel radiotherapy motion phantom using a stepper motor driver circuit and evaluation using optical surface scanning. Australasian Physical \& Engineering Sciences in Medicine, 40(1), 1-11.

[14] Viaene J. D., Haemers M., Verbelen F, et al. (2018) Current Reduction in Stepping Motor Applications using an Adaptive PI controller based on Linearized Dynamics Research funded by a PhD grant of the Research Foundation Flanders (FWO), Belgium. 51(4), 107-112.

[15] McQueney E., Landi M., Zhang L. (2017) Measurements of the Doppler effect due to a rotating sound source. Journal of the Acoustical Society of America, 142(4), 2542-2542. 